\title{
Effective Image Restorations Using a Novel Spatial Adaptive Prior
}

\author{
Yang Chen, ${ }^{1,2}$ Yinsheng Li, ${ }^{1,2}$ Yingmei Dong, ${ }^{3}$ Liwei Hao, ${ }^{2}$ Limin Luo, ${ }^{1}$ and Wufan Chen ${ }^{2}$ \\ ${ }^{1}$ The Laboratory of Image Science and Technology, Southeast University, Nanjing 210096, China \\ ${ }^{2}$ The School of Biomedical Engineering, Southern Medical University, Guangzhou 510515, China \\ ${ }^{3}$ Cadre Reset Institute, The Joint Logistics Department, Chengdu Military Region, Chengdu 610083, China \\ Correspondence should be addressed to Wufan Chen, chenwf@fimmu.edu.cn
}

Received 20 October 2009; Revised 29 December 2009; Accepted 16 February 2010

Academic Editor: Liang-Gee Chen

Copyright (๑) 2010 Yang Chen et al. This is an open access article distributed under the Creative Commons Attribution License, which permits unrestricted use, distribution, and reproduction in any medium, provided the original work is properly cited.

Bayesian or Maximum a posteriori (MAP) approaches can effectively overcome the ill-posed problems of image restoration or deconvolution through incorporating a priori image information. Many restoration methods, such as nonquadratic prior Bayesian restoration and total variation regularization, have been proposed with edge-preserving and noise-removing properties. However, these methods are often inefficient in restoring continuous variation region and suppressing block artifacts. To handle this, this paper proposes a Bayesian restoration approach with a novel spatial adaptive (SA) prior. Through selectively and adaptively incorporating the nonlocal image information into the SA prior model, the proposed method effectively suppress the negative disturbance from irrelevant neighbor pixels, and utilizes the positive regularization from the relevant ones. A twostep restoration algorithm for the proposed approach is also given. Comparative experimentation and analysis demonstrate that, bearing high-quality edge-preserving and noise-removing properties, the proposed restoration also has good deblocking property.

\section{Introduction}

1.1. Problem Formulation. As one of the most classical linear inverse problems, image restoration has its wide applications in remote sensing, radar imaging, tomographic imaging, microscopic imaging, astronomic imaging, digital photography, and so forth [1-4]. For linear and shiftinvariant imaging systems, the transformation from $f$ to $g$ is well described by following additive linear degradation model $[3,4]$ :

$$
g=A * f+\varepsilon,
$$

where $g, f$, and $\varepsilon$ represent, respectively, the degraded observed image, the original true image, and the corrupting white Gaussian noise with variance $\sigma^{2}$. Point-spread function (PSF) $A$ is the imaging system and $*$ is the linear convolution operator. Throughout this paper we assume that the degradation model PSF $A$ and noise variance $\sigma^{2}$ are known for they could be numerically estimated or calibrated.

Based on the Gaussian statistics of the additive noise, maximum log-likelihood (ML) restoration method could be applied to find the least-squares estimation of $f$. However, such ML restoration method often leads to unacceptable restoration solutions due to the ill-posedness of the inverse problems. Small changes in the data due to noise can cause large changes in the solution, and the knowledge of the degradation model is not sufficient to determine a restoration result with acceptable accuracy [2-5].

Bayesian or Maximum a posteriori (MAP) approach, within rigorous Markov random fields (MRFs) framework, can provide a stable regularized solution through the incorporation of a priori image information about the geometrical properties of an image [3-8]. Through modeling the unknown parameters in the prior probability density functions, such prior information measures the extent to which the contextual constraint assumption is violated by the image or surface. Bayesian approach is able to distinguish good solutions from less desirable ones by transforming the original ill-posed problem into a well-posed one. It is also noted that most regularization restoration approaches can find their Bayesian interpretation with different forms $[6,7]$. 
We can build following posterior probability $P(f \mid g)$ for image restoration

$$
\begin{gathered}
P(f \mid g) \propto P(g \mid f) P(f), \\
P(g \mid f)=\exp (L(g, f))=\exp \left(-\frac{1}{2} \kappa\|g-A * f\|^{2}\right), \\
P(f)=Z^{-1} \times \exp (-\alpha U(f))=Z^{-1} \times \exp \left(-\alpha \sum_{j} U_{j}(f)\right) .
\end{gathered}
$$

Here, $P(g \mid f)$ is the likelihood distribution and $P(f)$ is the prior distribution. The partition function $Z$ is a normalizing constant. $U(f)$ is the prior energy function, and $U_{j}(f)$ is the notation for the value of the energy function $U$ evaluated on the $f$ at pixel index $j . \alpha$ is the global hyperparameter that controls the degree of the prior's influence on the image $f$. The energy function $U(f)$ in (4) attains its minimum and the corresponding prior distribution (4) attains its maximum when the image meets the prior assumptions ideally. So from (2)-(4), we can build the log-posterior energy function as

$$
\begin{aligned}
\log P(f \mid g) & =L(g, f)-\alpha U(f) \\
& =-\frac{1}{2} \kappa\|g-A * f\|^{2}-\alpha \sum_{j} U_{j}(f) .
\end{aligned}
$$

The reconstructed image $f$ can be obtained through iteratively maximizing function $\psi(f)$.

The simple and widely used quadratic membrane (QM) prior or the Tikhonov L2 regularization, which smoothes both noise and edge details equally, leads to a linear inversion process and tends to produce an unfavorable oversmoothing effect [5].

To solve this oversmoothing problem, many edgepreserving Bayesian restoration methods were proposed in the past twenty years. We generalize them into three main classes: wavelet Bayesian restoration, Bayesian restoration with nonquadratic prior energies, and Bayesian restoration with total variation (TV) prior/regularization.

Wavelet complexity regularization restorations, using a multiscale stochastic prior model, have also been proposed for edge-preserving restoration [8-11]. Priors based on wavelet decompositions and heavy-tailed pdfs along with the EM restoration algorithm have been proposed in [10]. These wavelet regularization methods rely on statistical modeling of the distribution of wavelet coefficients and strategy of coefficient thresholding. We choose not to include the comparison of wavelet regularization methods in this paper for the consideration that they can also be reinterpreted as some wavelet domain forms for Wiener filters, TV variational methods, or some Tikhonov regularization procedures [11].

Edge-preserving priors with nonquadratic energies were also proposed to preserve edge details by assuming a nonlinear inverse-proportional relationship between the existence of edges and intensity differences $[5,12,13]$. The weighting matrices in nonquadratic prior Bayesian restoration preserve edges by turning off or suppressing smoothing at appropriate locations.
Another impressive recent advance in this area is the total variation- (TV-) based image restoration algorithms $[7,14,15]$. This kind of approach can also be viewed as TV prior with the half-quadratic regularization prior energy. The inherent idea of TV methods describes the target images as consisting largely of zero-gradient regions interspersed with occasional strong gradient transitions. With no enforced global image continuity, TV prior restorations often demonstrate good edge-preserving property.

The nonquadratic priors aim to preserve edges through determining the regularization degree on each pixel based on the intensity-difference information within a local fixed neighborhood. The regularization information of TV prior also comes from the local neighboring intensity gradients. Limited by local prior information within local neighborhoods, these edge-preserving approaches cannot cope well with realistic complicated images, and tend to produce blocky artifacts around continuous edges [16-21].

1.2. Existing Solution and Our Proposed One. A more efficient edge-preserving restoration model should be more adaptive to the characteristic structure of the image to avoid or suppress the unfavorable blocky artifacts. In the past twenty years some spatially adaptive techniques were proposed to in this way.

In this way, a line-process [5] or doubly stochastic MRF model [22] has been proposed. Bouman and Sauer proposed a generalized Gaussian MRF prior model with a power parameter controlling the background smoothness and the edge sharpness in the restored image. However, using line elements adds to the complexity of the problem by increasing both the dimensionality of the required optimization and the complexity of the parameter estimation procedure.

In [16], Mignotte proposed an adaptive region-based edge-preserving regularization term, which applies a local smoothness constraint to the preestimated constant-valued regions by a two-level MRF model. The first level is a lowlevel prior model used in the segmentation procedure, and the second prior model exploits the segmentation result (a partition into regions extracted from this segmentation map) as a parameter for the resulting restoration. And in a similar way but with a different application, Yu and Fessler devised a boundary-based Bayesian method for transmission tomography after level-set segmentation [23]. Such boundary or region-based methods rely heavily on the segmentation operations whose effect in different images is unpredictable and parameter dependent.

And recently in [18], Chantas et al. proposed a new hierarchical (two-level) Gaussian nonstationary image prior with assumption that the residuals of the first-order directional differences of the image are spatially varying Gaussian random variables. The local directional variances of these differences are utilized to capture the discontinuities. The two main drawbacks of this nonstationary approach are that the normalization constant for the prior is heuristically adjusted and also the hyperparameters need to be found heuristically. 
In this article, based on the work in [24] and our previous tomographic work [19-21], we propose a spatial adaptive Bayesian restoration approach. This new restoration approach utilizes a spatial adaptive (SA) prior, which exploits the global connectivity and continuity information in the objective image. It works by adaptively including the useful relevant neighbor pixels and excluding the negative irrelevant ones within a large prior neighborhood. The proposed restoration has good noise-removing and edge-preserving properties, and, especially, it can effectively restore continuous regions with much less blocky artifacts.

In Section 2, a review of the conventional prior model and the theory for the proposed SA prior model are both illustrated. In Section 3, we give the iterative restoration algorithm for the proposed approach. In Section 4, we perform restoration using different methods under the programming environment of hybrid Matlab and $\mathrm{C}$ language. Relevant visual and quantitative comparisons are also shown. Conclusions and plans for future work are given in Section 5.

\section{Prior Model}

In this section, after providing a review of the conventional prior model, we introduce the theory for the proposed SA prior model.

2.1. The Traditional Prior Model. Conventionally, the value of $U_{j}(f)$ is commonly computed through a weighted sum of potential functions $v$ of the differences between pixels in the neighborhood $N_{j}$ :

$$
U_{j}(f)=\sum_{b \in N_{j}} w_{b j} v\left(f_{b}-f_{j}\right) .
$$

Generally, different choices of the potential function $v$ lead to different priors. The prior is the familiar spaceinvariant QM prior when the potential function has the form $v(t)=t^{2} / 2$.

Edge-preserving nonquadratic priors could be chosen by adopting a nonquadratic potential function for $v$, such as the Huber potential function:

$$
v(t)= \begin{cases}\frac{t^{2}}{2}, & |t| \leq \gamma, \\ \gamma|t|-\frac{\gamma^{2}}{2}, & |t|>\gamma,\end{cases}
$$

where $\gamma$ is the threshold parameter $[5,13,16]$. Such edgepreserving nonquadratic prior preserves the edge information by choosing a nonquadratic potential function that increases less as the differences between adjacent pixels become bigger. Weight $w_{b j}$ is a positive value that denotes the interaction degree between pixel $b$ and pixel $j$. And in traditional prior models, it is simply considered to be inversely proportional to the distance between pixel $b$ and pixel $j$. So on a square lattice of image $f$, in which the $2 \mathrm{D}$ positions of the pixel $b$ and pixel $j(b \neq j)$ are, respectively, $\left(b_{x}, b_{y}\right)$ and $\left(j_{x}, j_{y}\right), w_{b j}$ is usually calculated by the geometric distance $1 / \sqrt{\left(b_{x}-j_{x}\right)^{2}+\left(b_{y}-j_{y}\right)^{2}}$ or some other simple forms. Here, for traditional prior models, we illustrate two widely used normalized weighting maps for eight-element neighborhood and four-element neighborhood:

(1) $(1 /(4 \times 1+4 \times 1 / \sqrt{2})) \times \begin{array}{ccc}1 / \sqrt{2} & 1 & 1 / \sqrt{2} \\ 1 & 0 & 1 \\ 1 / \sqrt{2} & 1 & 1 / \sqrt{2}\end{array}$

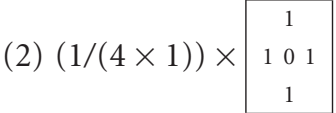

Restorations using Total Variation (TV) prior or regularization often take prior energy as follows:

$$
U_{\mathrm{TV}}(f)=\sum_{j} \sqrt{\left(\Delta_{j}^{h} f\right)^{2}+\left(\Delta_{j}^{v} f\right)^{2}}
$$

where $\Delta_{j}^{h}$ and $\Delta_{j}^{v}$ are linear operators corresponding to horizontal and vertical first-order differences at pixel $j$, respectively $[7,14,15]$. This TV prior energy given by $(8)$ favors images with bounded variation without penalizing possible discontinuities since both smooth and sharp edges have the same TV prior energy.

2.2. The Proposed SA Prior Model. Objects in most images have edges with coherently varying pixel intensities. Pixelscale intensity differences alone are insufficient to characterize objects of multiple scales fully. Traditional quadratic priors, nonquadratic priors, and TV prior can only provide fixed local information for image restoration. Through an averaging alike effect the local QM prior or Tikhonov regularization is prone to produce an unfavorable oversmoothing effect to smooth out both noise and details. And the edge-preserving nonquadratic and TV priors, although able to preserve some edge information, tend to produce unfavorable blocky artifacts or false edges because of the disturbance of noise. In [22], we find that simply enlarging the prior neighborhood of local prior is ineffective in improving restoration.

Thus continuous texture information within a larger scale should be used to discriminate image information from singularities or noise. This laid the basis of the proposed method. In the building of the proposed SA prior model, a large nonlocal neighborhood $N$ is used to incorporate geometrical configuration information. Other than calculated as the Gaussian decreasing function of the difference between the two square comparing neighborhoods as the methods in [19], the weight $w_{b j}$ for the new selective nonlocal prior is set to be a 1-0 binary function which classifies all pixels in neighborhood $N$ into two classes-the neighbors relevant to the center pixel $j$ and those not. Only the relevant ones are picked out for the SA prior. The value of $w_{b j}$ is set to be 0 or 1 when the computed distance $d_{b j}$ is greater or smaller than a set threshold $\delta$.

Thus, the chosen relevant pixels in the SA prior neighborhood for each center pixel $j$ are not fixed. Both the numbers and positions of the relevant pixels in neighborhood $N$ are adjusted spatially and adaptively with the nonstationary 


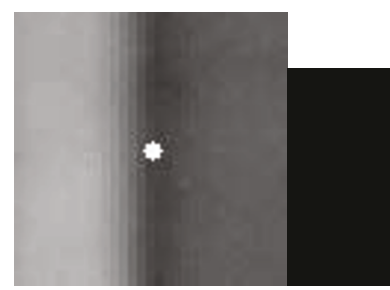

(a)
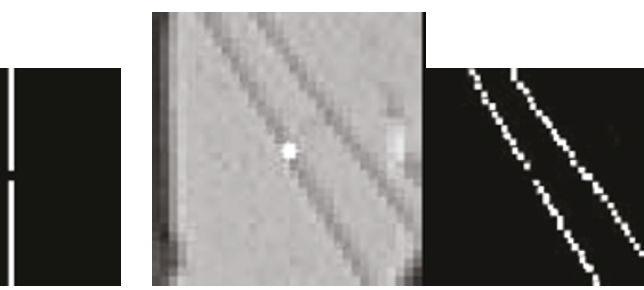

(b)

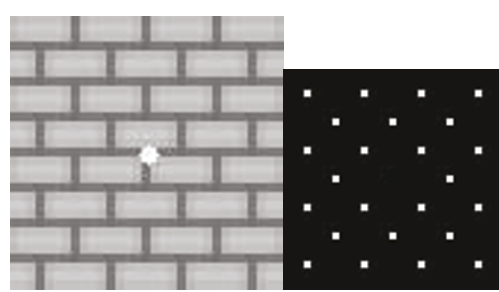

(c)
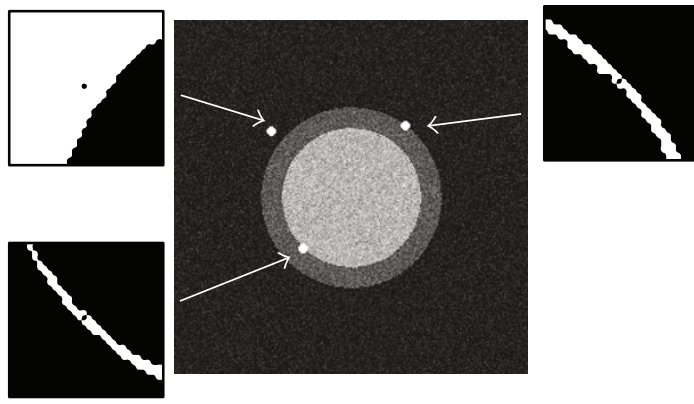

(d)

FIGURE 1: Illustrations of the weight distributions of the adaptive neighborhood system for the proposed SA prior model. The sizes of neighborhoods $N$ and $n$ are $31 \times 31$ and $7 \times 7$, respectively. (a) The weight factors $w_{b j}$ are distributed in the direction of the straight edge line when the centered pixel $j$ is in a straight alike configuration. (b) The weight factors $w_{b j}$ are distributed in the direction of the edge line when the centered pixel $j$ is in an oblique edge. (c) The weight factors $w_{b j}$ are adaptively distributed along the pixels in the same structures when the centered pixel $j$ is in some special structure. (d) The weight factor distributions for the three centered pixels in the two edge regions and the background region, respectively, are shown.

spatial properties of the objective image. The building of the proposed SA prior can be formalized as follows:

$$
\begin{gathered}
U_{\mathrm{SA}}(f)=\sum_{j} U_{j}(f)=\sum_{j} \sum_{b \in N_{j}}\left(w_{b j}\left(f_{b}-f_{j}\right)^{2} /\left|N r_{j}\right|\right), \\
w_{b j}= \begin{cases}1, & \operatorname{dis}_{b j} \leq \delta \\
0, & \operatorname{dis}_{b j}>\delta\end{cases} \\
\operatorname{dis}_{b j}=\left\|n_{b}(f)-n_{j}(f)\right\|_{E}^{2}=\sum_{l}\left(f_{l \in n_{b}}-f_{l \in n_{j}}\right)^{2}, \\
n_{b}(f)=\left\{f_{l}: l \in n_{b}\right\} \\
n_{j}(f)=\left\{f_{l}: l \in n_{j}\right\} .
\end{gathered}
$$

Here, $U_{\mathrm{SA}}$ is the energy function for the proposed SA prior. $w_{b j}$, which represents the classification of the neighbor pixels in the search neighborhood $N_{j}$, can be computed via (10)(13). $\left|N r_{j}\right|$, the number of neighbor pixels with nonzero $w_{b j}$ in the neighborhood $N_{j}$, can function as a normalized factor of hyperparameter $\beta$ for the different numbers of relevant neighbor pixels in each search neighborhood $N_{j}$. Parameter $\delta$ in (10) is the threshold parameter. The value of the distance dis $_{b j}$ is determined by a distance measurement between the two translated comparing neighborhoods $n_{b}$ and $n_{j}$. The two comparing neighborhoods $n_{b}$ and $n_{j}$ have same sizes and are centered at pixel $b$ and pixel $j . n_{b}$ and $n_{j}$ can also be considered two $2 \mathrm{D}$ pixel intensity vectors including all inner pixels of neighborhoods $n_{b}$ and $n_{j} . l$ is the index denoting the two pixels with the same positions related to the corresponding center pixel $b$ and pixel $j$ in $n_{b}$ and $n_{j}$, respectively.

Through the computation and thresholding of the distances between the comparing neighborhoods $n$ centered on the corresponding pairs of pixels in the large neighborhood $N$, the spatial-varying weights of each pair of pixels in $N$ are distributed across the similar configurations. Thus global configuration information can be exploited with the negative disturbance from the irrelevant pixels suppressed.

To make above statement of the spatial adaptive neighborhood model more straightforward and clearer, in Figure 1 we illustrate several simulated weight factors distribution maps of the tagged pixels for the nonlocal prior. In all illustrations, the sizes of neighborhoods $N$ and $n$ are set to be $31 \times 31$ and $7 \times 7$, respectively. In Figure 1(a), we can see that the prior weight factors are distributed along the direction of the straight edge line in neighborhood $N$ when the pixel is in a straight edge. In Figure 1(b), when the pixel belongs to an oblique edge, the corresponding prior weight factors are distributed along the same oblique edge in neighborhood $N$. In Figure 1(c), the corresponding prior weight factors are distributed along the same structures in neighborhood $N$ when the pixel belongs to one specific structure. Figure 1(d) illustrates three prior weight distributions for the three pixels in edge regions and background region, respectively. We can also see that the SA prior weight factors for the two pixels in edge regions are adaptively distributed along the edge regions, while the prior weight factors for the pixel 
in background region are distributed in the corresponding background region.

Compared to the Gaussian weighting form in [19, 24], this new binary weighting approach, which explicitly classifies all pixels in neighborhood $N$, is observed to produce similar results after optimal parameter settings. Notably, this binary weighting approach has more straightforward relating from $d_{b j}$ to $w_{b j}$, which permits more tractable setting of threshold $\delta$. It can also reduce computation cost by avoiding calculation of exponential functions in the Gaussian weighting form.

\section{Restoration Algorithm}

From (5), Bayesian restoration with the proposed SA prior model can thus be defined as the search of the global minima of the following energy function (14) with respect to $f$ :

$$
\psi_{\beta}(f \mid g)=\|g-A * f\|^{2}+\beta U_{\mathrm{SA}}(f) .
$$

Here the energy function contains a fidelity term $\|g-A * f\|^{2}$ and a prior energy term $U_{\mathrm{SA}} \beta=\alpha / \kappa$ is the regularization parameter controlling the tradeoff of the two terms.

Obviously the fidelity term $\|g-A * f\|^{2}$ given by (14) is quadratic and convex. Considering the convexity for quadratic function $\left(f_{b}-f_{j}\right)^{2}$ in (9), (14) is definitely convex if the values of all $w_{b j}$ are fixed. With an assumption of fixed $w_{b j}$, convergent estimation updates toward global minima can be easily obtained using Newton-Raphson algorithm $[25,26]$. However, from (9)-(13), we know that every weighting factor $w_{b j}$ is not fixed and needs to be determined by the estimated image $\hat{f}$ each iteration, which makes it difficult to preserve the global convergence of above NewtonRaphson iteration. A practical suboptimal alternating twostep restoration algorithm is as follows. After choosing an initial image estimate $f^{0}$, we can update weight $w$ and image $f$ alternatively using following two-step strategy for every iteration.

(1) Weight Update. For every pixel pair $\left(f_{b}, f_{j}\right)$ in image $f$ with $\hat{f}^{n}$ being the estimate of $f$ from the $n$th iteration, compute $w_{b j}$ using (9)-(13) and $\hat{f}^{n}$.

(2) Image Update. We can obtain following closed-form updating solution using the Newton-Raphson algorithm:

$$
\begin{aligned}
\hat{f}^{n+1}=\hat{f}^{n}+\left(\left.\frac{\partial}{\partial f} \psi_{\beta}(f \mid g)\right|_{f=\hat{f}^{n}}\right) & \\
& /\left(-\left.\frac{\partial^{2}}{\partial f^{2}} \psi_{\beta}(f \mid g)\right|_{f=\hat{f}^{n}}\right), \\
\hat{f}^{n+1}= & \hat{f}^{n}+\left.\left(A^{T}\left(g-A * f^{n}\right)-\beta \frac{\partial}{\partial f} U_{\mathrm{SA}}(f)\right)\right|_{f=\hat{f}^{n}} \\
& /\left.\left(A^{T} A+\beta \frac{\partial^{2}}{\partial f^{2}} U_{\mathrm{SA}}(f)\right)\right|_{f=\hat{f}^{n}} \cdot
\end{aligned}
$$

Coordinate Descent (CD) method, which updates one pixel at a time using the most recent other pixels, can be combined with this Newton-Raphson algorithm to increase convergence [26]. Such joint updating restoration algorithm can be considered as a version of the widely used OSL (One-Step-Late) iterative algorithm $[27,28]$. Though able to converge to at least a local minimum as the joint algorithm proposed in [23], this joint updating restoration algorithm suffers from the lack of strict convergence proof.

To obtain a convergent iteration process when using the proposed prior with changing $w_{b j}$ that has to be determined by current estimate $\hat{f}$, we choose to fix the values of all $w_{b j}$ after some iterations. Thus after some above nonconvergent OSL Newton-Raphson iterations with variable weighting factors $w_{b j}$, we fix all $w_{b j}$ and monotonically minimize the energy function $\psi_{\beta}(f \mid g)$ using the Newton-Raphson algorithm (Image Update). So, in practical experiments, we set a fixed iteration number $\Gamma$ and apply this updating strategy to obtain a global solution for the Bayesian restoration using the proposed prior.

Threshold parameter $\delta$ in (10) cannot be estimated exactly, and we manually set the value of $\delta$ using the dis (computed by formula (11)) information of the image in terms of signal-to-noise ratio (SNR) measurements and visual performance. SNR can be calculated as follows:

$$
\mathrm{SNR}=10 \log _{10}\left(\frac{\sum_{j}^{M}\left(f_{j}-\bar{f}\right)^{2}}{\sum_{j}^{M}\left(f_{j}-\hat{f}_{j}\right)^{2}}\right)
$$

Here $M, f, \bar{f}$, and $\hat{f}$ denote the total pixel number, the original image, mean of the original image, and the restored image, respectively.

One drawback of the proposed approach is the heavy computation burden due to its complexity in calculating all the inter weight $w$ between each two translated comparing neighborhoods $n$ in search neighborhood $N$ over the whole image region. In practical, we generally do not extend the neighborhood bounds over the whole images, and choose suitable $7 \times 7$ neighborhood $N$ and $7 \times 7 n$, which is proved to be large enough to provide effective regularization in Section 4.2. And, considering the symmetry property that the distance $\operatorname{dis}_{b j}$ equals the distance $\operatorname{dis}_{j b}$, we can save one half computation cost by only calculating one of the two distances $\operatorname{dis}_{b j}$ and $\operatorname{dis}_{j b}$. Furthermore, considering the inefficiency of cycling operation under Matlab7.5 environment, all of the Matlab codes for weight calculation are transformed to mixed $\mathrm{C}$ language. By applying above methods, we successfully lowered the CPU computational costs to around $1 / 4$ of the original costs.

\section{Numerical Experiments}

In this section, we present simulated experiments with numerical comparisons. The two metrics used to quantify the quality of the degraded images, the noise levels in our degraded images, and the quality of different restoration 
TABLE 1: Image and degradation information for the three test images.

\begin{tabular}{lccc}
\hline Test images $(256 \times 256)$ & Intensity ranges & Simulated PSFs & Noise properties \\
\hline "Lena" & {$[-98-117]$} & Uniform 9 $\times 9$ PSF & Gaussian noise $(\sigma=0.1663)$ \\
"Sailing Boat" & {$[-123-129]$} & Uniform 7 $\times 7$ PSF & Gaussian noise $(\sigma=0.3511)$ \\
"Parrot" & {$[-73-148]$} & Uniform 7 $\times 7$ PSF & Gaussian noise $(\sigma=0.3740)$ \\
\hline
\end{tabular}

TABLE 2: Optimal parameter settings.

\begin{tabular}{lccc}
\hline Test images & TV prior & Huber prior & SA prior \\
\hline "Lena" & $\beta=0.0038$ & $\beta=0.026, \gamma=0.35$ & $\beta=0.015, \delta=900$ \\
"Sailing Boat" & $\beta=0.006$ & $\beta=0.044, \gamma=0.45$ & $\beta=0.015, \delta=800$ \\
"Parrot" & $\beta=0.0085$ & $\beta=0.025, \gamma=0.55$ & $\beta=0.02, \delta=350$ \\
\hline
\end{tabular}

results are the SNR and the improvement in signal-to-noise ratio (ISNR). ISNR is defined as

$$
\operatorname{ISNR}=10 \log _{10}\left(\frac{\sum_{j}^{M}\left(f_{j}-g_{j}\right)^{2}}{\sum_{j}^{M}\left(f_{j}-\hat{f}_{j}\right)^{2}}\right) .
$$

Here $M, f, \hat{f}$, and $g$ denote the total pixel number, the original image, the restored image, and the observed degraded image, respectively.

Restoration experiments with three $256 \times 256$ images ("Lena" (Figure 2(a)), "Sailing Boat" (Figure 3(a)), and "Parrot" (Figure $4(\mathrm{a})$ ) are performed. Image and degradation information is listed in Table 1. The three PSFs used in experiment are all normalized to 1 . Observed degraded images (resp., in Figures 2(b), 3(b), and 4(b)) were simulated by (1) using corresponding PSFs and white Gaussian noise. All of the restorations using the three images are shown in Figures 2, 3, and 4, respectively.

4.1. Results from Different Restoration Methods. In order to compare the proposed approaches with previous ones, we implement Wiener filter restoration, Bayesian restoration using TV prior, and Huber prior. We perform the classical Wiener filter in the DFT domain using the degraded image to estimate the image power spectrum and assuming that the additive noise variance is known. The resulting images are, respectively, shown in Figures 2(c), 3(c), and 4(c).

The hyperparameter $\beta$ for all Bayesian restorations, threshold parameter $\gamma$ for Huber prior, and threshold parameter $\delta$ for the nonlocal prior are chosen manually using the SNR criteria. The used parameters for different restorations are listed in Table 2.

The TWIST [7] and Newton-Raphson algorithm are used in restorations using TV prior and Huber prior, respectively. Restored images from above Wiener filter method are used as the initial images. The iteration number is set to be 500 . The corresponding restored images from TV prior restoration are, respectively, shown in Figures 2(d), 3(d), and 4(d). And the corresponding restored images from Huber prior restoration are, respectively, shown in Figures 2(e), 3(e), and $4(\mathrm{e})$.
TABLE 3: Signal-to-noise ratio (SNR) of the observed degraded images and the restored images.

\begin{tabular}{lccccc}
\hline Test images & $\begin{array}{c}\text { Degraded } \\
\text { image }\end{array}$ & $\begin{array}{c}\text { Wiener } \\
\text { filter }\end{array}$ & $\begin{array}{c}\text { TV } \\
\text { prior }\end{array}$ & $\begin{array}{c}\text { Huber } \\
\text { prior }\end{array}$ & SA prior \\
\hline "Lena" & 6.65 & 15.06 & 15.54 & 15.59 & 15.86 \\
"Sailing Boat" & 6.97 & 15.76 & 16.43 & 16.45 & 16.91 \\
"parrot" & 7.98 & 16.75 & 17.25 & 17.22 & 17.86 \\
\hline
\end{tabular}

TABLE 4: Improvement in signal-to-noise ratio (ISNR) of the observed degraded images and the restored images.

\begin{tabular}{lcccc}
\hline Test Images & Wiener filter & TV prior & Huber Prior & SA prior \\
\hline "Lena" & 5.79 & 8.48 & 8.76 & 9.15 \\
"Sailing Boat" & 7.12 & 10.10 & 10.54 & 11.13 \\
"Parrot" & 8.06 & 11.45 & 11.99 & 12.40 \\
\hline
\end{tabular}

For Bayesian restoration using the proposed SA prior, the $7 \times 7$ neighborhood $N$ and the $7 \times 7$ comparing neighborhood $n$ are used. We apply above two-step Newton-Raphson algorithm with a prefixed iteration number $\Gamma$. Restored images from the fifth TV iteration are chosen as the initial images to achieve faster convergence. The total iteration number is set to be 500 with $\Gamma=200$. And the corresponding restored images are, respectively, shown in Figures 2(f), 3(f), and 4 (f).

Through comparison, we find that all of the three Bayesian restorations can effectively overcome the annoying ring effects in Wiener filter restoration. Especially, the block artifacts, which can be observed in the Bayesian approach using Huber prior and TV prior, are effectively suppressed using the proposed method. Restorations using the proposed SA prior perform better in both noise suppressing and edge preserving. Using the proposed approach, continuous regions and details are better restored with much less block artifacts produced. We can also see in Tables 3 and 4 that the proposed SA prior restoration approaches can restore images with higher SNR and ISNR values.

4.2. Study on Neighborhoods $N$ and $n$ of Different Sizes. To find out the impacts of the sizes of neighborhoods $N$ and $n$ 


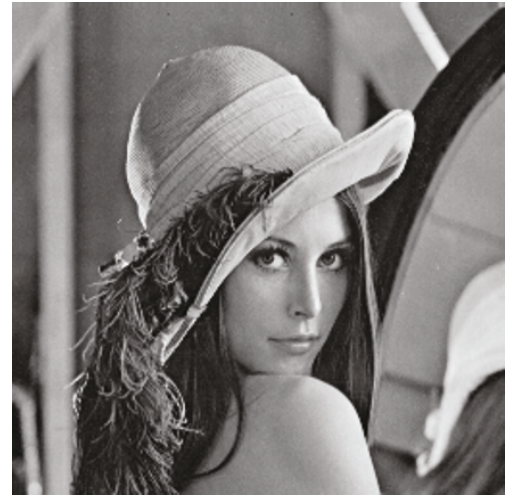

(a)

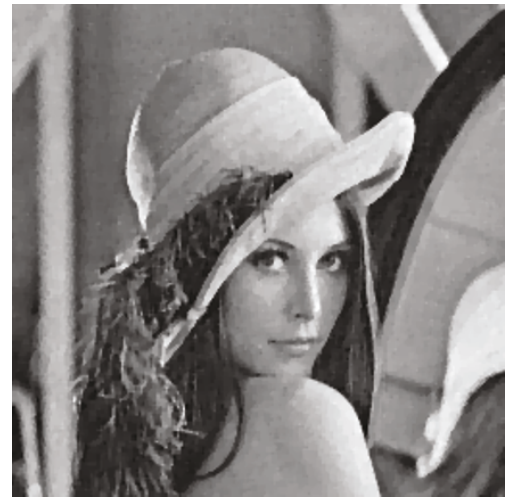

(d)

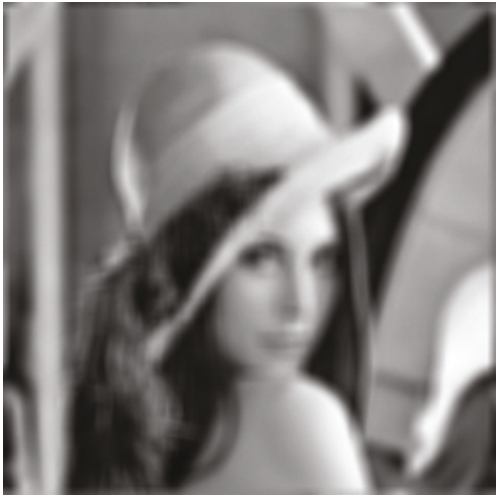

(b)

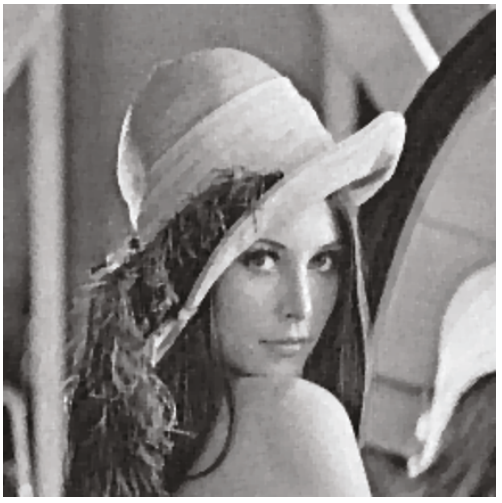

(e)

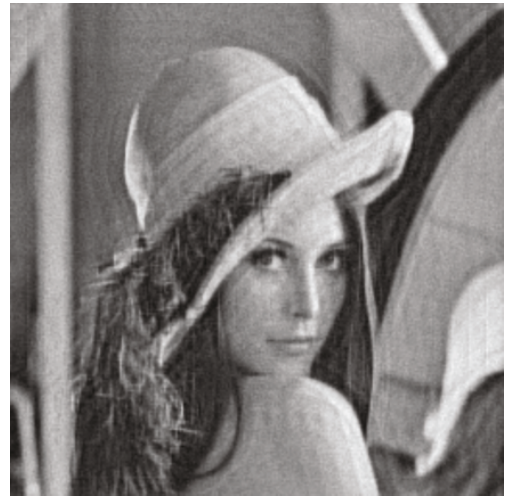

(c)

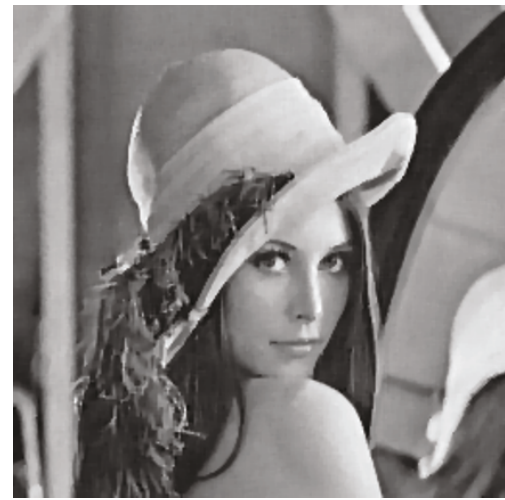

(f)

FIgURE 2: (a) Original "Lena" image. (b) Degraded "Lena" image (SNR $=6.65)$ with $9 \times 9$ uniform blur and additive white Gaussian noise (local variance $=0.1663$ ). (c) Wiener filter restoration. (d) TV prior Bayesian restoration. (e) Nonquadratic Huber prior Bayesian restoration. (f) The proposed SA prior Bayesian restoration.

TABle 5: Combinations of neighborhoods $N$ and $n$ with different sizes.

\begin{tabular}{lll}
\hline$a, N_{3 \times 3} n_{1 \times 1}$ & $b, N_{3 \times 3} n_{3 \times 3}$ & $c, N_{3 \times 3} n_{7 \times 7}$ \\
$d, N_{7 \times 7} n_{1 \times 1}$ & $e, N_{7 \times 7} n_{3 \times 3}$ & $f, N_{7 \times 7} n_{7 \times 7}$ \\
$g, N_{11 \times 11} n_{1 \times 1}$ & $h, N_{11 \times 11} n_{3 \times 3}$ & $i, N_{11 \times 11} n_{7 \times 7}$ \\
\hline
\end{tabular}

TABLE 6: SNR for the restorations using the proposed SA prior with the corresponding neighborhood combinations in Table 5.

\begin{tabular}{lll}
\hline$a, N_{3 \times 3} n_{1 \times 1}: 15.55$ & $b, N_{3 \times 3} n_{3 \times 3}: 15.68$ & $c, N_{3 \times 3} n_{7 \times 7}: 15.60$ \\
$d, N_{7 \times 7} n_{1 \times 1}: 15.53$ & $e, N_{7 \times 7} n_{3 \times 3}: 15.72$ & $f, N_{7 \times 7} n_{7 \times 7}: 15.86$ \\
$g, N_{11 \times 11} n_{1 \times 1}: 15.43$ & $h, N_{11 \times 11} n_{3 \times 3}: 15.69$ & $i, N_{11 \times 11} n_{7 \times 7}: 15.89$ \\
\hline
\end{tabular}

in restoration, we perform restorations of $256 \times 256$ image "Lena" using the proposed SA prior with neighborhoods $N$ and $n$ of different sizes. The total iteration number is set to be 500 with $\Gamma=200$. Same restoration environment as above is used. The combinations of neighborhoods $N$ and $n$ with different sizes are illustrated in Table 5.

Considering the fact that the numbers of pixels are different for different comparing neighborhoods $n$ (49 for $7 \times 7$ neighborhood $n, 9$ for $3 \times 3$ neighborhood $n$, and 1 for $1 \times 1$ neighborhood $n$ ), parameter $\delta$ should also change with restorations using different comparing neighborhoods $n$. The values of parameter $\delta$ for $7 \times 7 n, 3 \times 3 n$, and $1 \times 1 n$ are set to be 900,400 , and 125 , respectively. The hyperparameter $\beta$ for the SA prior is chosen by hand under the same strategy in terms of the SNR criteria.

We can see in Table 6 that higher SNR can be obtained for larger $N$ and $n$. When choosing $1 \times 1 n$ with $N$ of different sizes, the comparing neighborhoods $n$ become one singlepixel and only single pixel intensities are compared in (11). In this situation the SA prior becomes a modified version of local Huber prior. Choosing $7 \times 7 N$ with $7 \times 7 n$, we can restore the images with almost the same SNR as those with larger $N . N$ and $n$ of a suitable size $(7 \times 7$ for both in this situation) might be large enough to achieve good restorations.

We also list in Table 7 the CPU time costs for above restorations using the proposed prior with above different combinations of neighborhoods. The methods for computation reduction in Section 3 are used. As predicted, more CPU time costs are needed for the restorations using the proposed prior with larger $N$ and $n$.

4.3. Iteration Monotonicity. We know from the analysis in Section 3 that the Hessian matrix of the $\psi_{\beta}(f \mid g)$ in (14) is positive definite in each single iteration if the values 


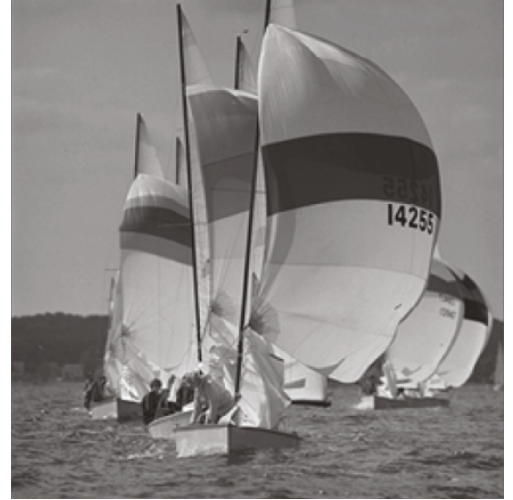

(a)

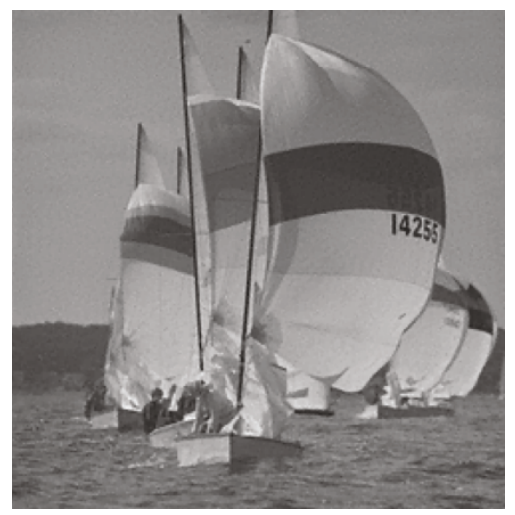

(d)

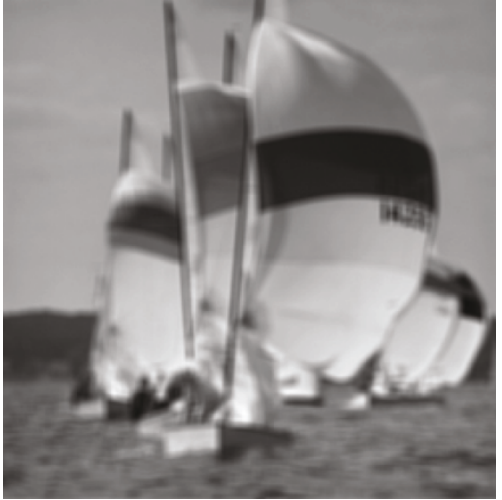

(b)

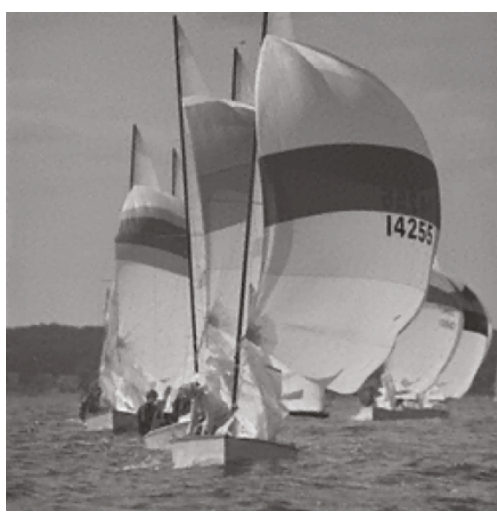

(e)

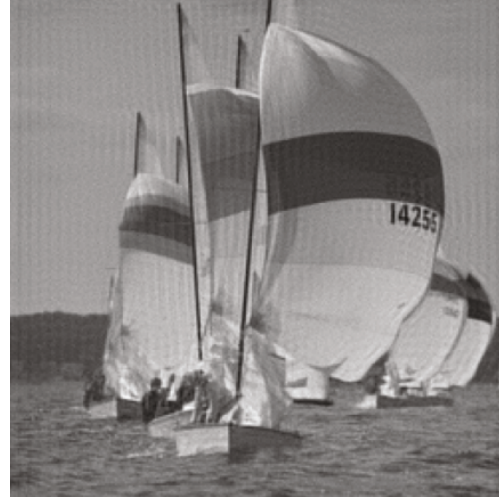

(c)

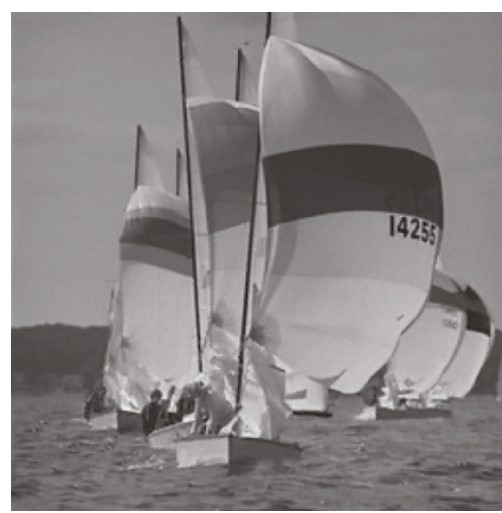

(f)

FIGURE 3: (a) Original "Sailing Boat" image. (b) Degraded "Sailing Boat" image (SNR $=6.97$ ) with $7 \times 7$ uniform blur and additive white Gaussian noise (local variance $=0.3511$ ). (c) Wiener filter restoration. (d) TV prior Bayesian restoration. (e) Nonquadratic Huber prior Bayesian restoration. (f) The proposed SA prior Bayesian restoration.

TABLE 7: CPU times in terms of seconds needed for above restorations using the proposed SA prior with the corresponding neighborhood combinations in Table 5.

\begin{tabular}{lll}
\hline$a, N_{3 \times 3} n_{1 \times 1}: 58.16 \mathrm{sec}$ & $b, N_{3 \times 3} n_{3 \times 3}: 96.84 \mathrm{sec}$ & $c, N_{3 \times 3} n_{7 \times 7}: 150.72 \mathrm{sec}$ \\
$d, N_{7 \times 7} n_{1 \times 1}: 73.72 \mathrm{sec}$ & $e, N_{7 \times 7} n_{3 \times 3}: 118.30 \mathrm{sec}$ & $f, N_{7 \times 7} n_{7 \times 7}: 224.60 \mathrm{sec}$ \\
$g, N_{11 \times 11} n_{1 \times 1}: 97.62 \mathrm{sec}$ & $h, N_{11 \times 11} n_{3 \times 3}: 148.78 \mathrm{sec}$ & $i, N_{11 \times 11} n_{7 \times 7}: 321.48 \mathrm{sec}$ \\
\hline
\end{tabular}

of all $w_{b j}$ are fixed. However, before preset $\Gamma$ iterations, no strict convergence is guaranteed because the posterior energy function changes with the weighting factors which are needed to be updated in each iteration. Here, in Figure 5, we calculate and plot the posterior function energy $\psi_{\beta}(f \mid$ $g$ ) with respect to iteration numbers for above restoration experiment with "Lena" image. The total iteration number is set to be 1000 with $\Gamma=200$ to include a long iteration process. We can see that the calculated posterior function energy decreases monotonically during the whole iteration process.

We thus conclude that, using the proposed algorithm, monotonic decrement of the posterior function energy can be achieved after $\Gamma$ iterations when the weighting factors begin to be fixed and fixed posterior energy function is guaranteed.

\section{Conclusions and Future Work Plan}

In this paper, based on MRF theory and our previous work, we proposed a novel spatial adaptive prior Bayesian restoration approach. The proposed method can incorporate the global connectivity information into image restoration. Not only capable of suppressing background noise and preserving details, the proposed restoration is also capable of restoring continuous regions and suppressing blocky artifacts effectively. An SNR performance comparisons of restorations using the proposed prior with different combinations of neighborhoods $N$ and $n$ are also illustrated. We find that good reconstructions can be obtained with neighborhood sizes suitably chosen. Compared to the methods in [16-18], the proposed SA prior model is less complicated and needs no additional segmentation alike empirical operation. 


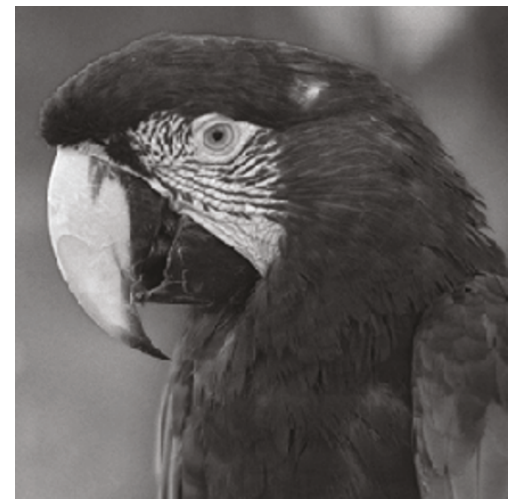

(a)

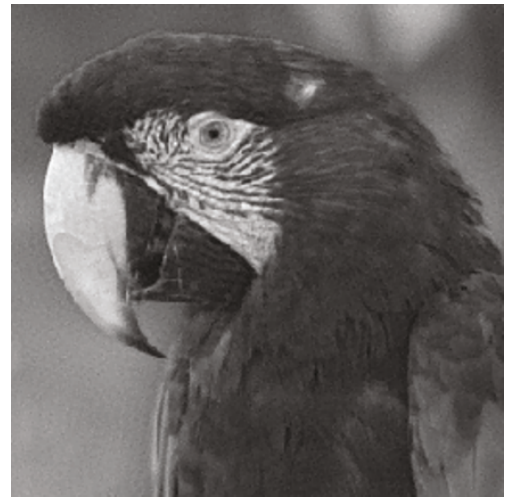

(d)

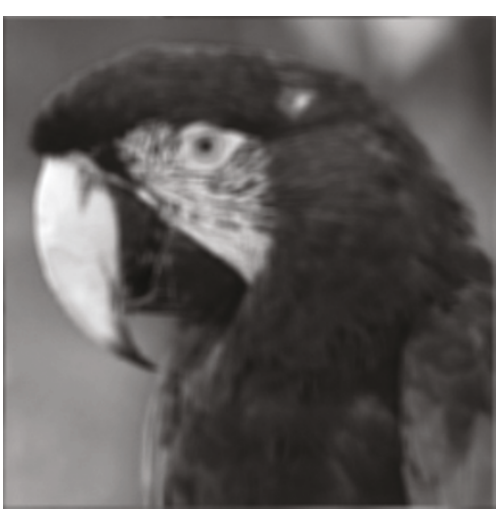

(b)

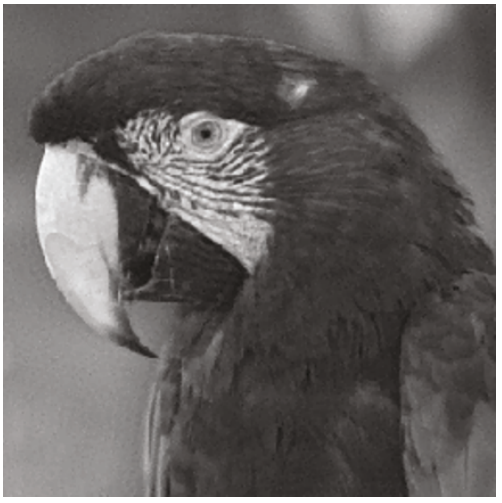

(e)

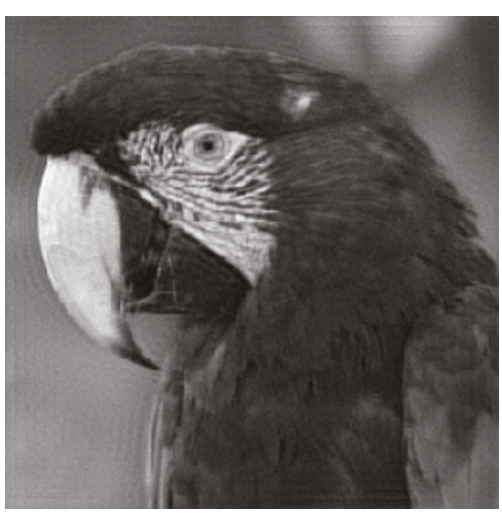

(c)

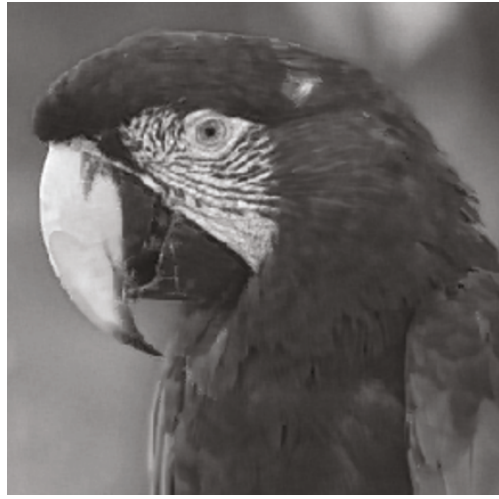

(f)

FIgure 4: (a) Original "Parrot" image. (b) Degraded "Parrot" image (SNR $=7.98$ ) with $7 \times 7$ uniform blur and additive white Gaussian noise (local variance $=0.3740$ ). (c) Optimal Wiener filter restoration. (d) TV prior Bayesian restoration. (e) Nonquadratic Huber prior Bayesian restoration. (f) The proposed SA prior Bayesian restoration.

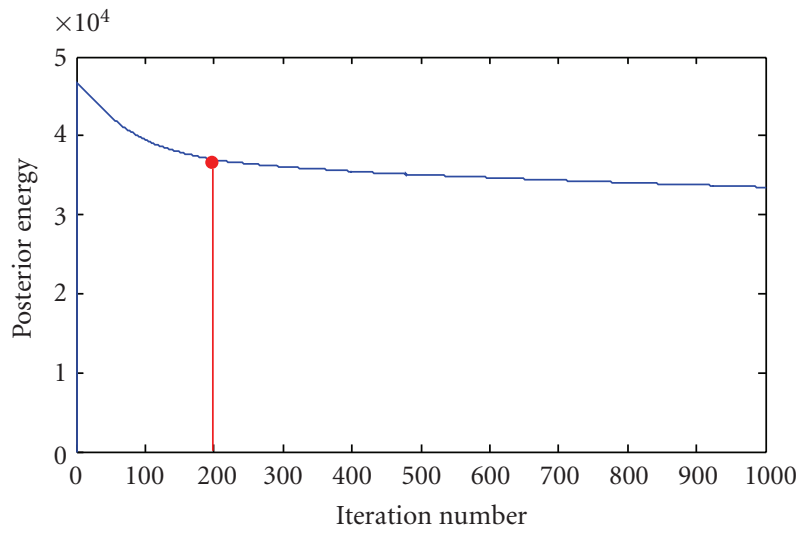

FIGURE 5: Using the proposed restoration algorithm in Section 3, the calculated posterior function energy with respect to iteration number is shown.

Nevertheless, although several computation reduction approaches have been applied, the proposed approach still needs computations over large neighborhoods and estimations of hand-adjusted parameters (threshold $\delta$ and the sizes for neighborhoods $N$ and $n$ ), which make it high computational cost compared to other methods. However, considering the ever-increasing computing power and the need for high-quality restoration, the increased computation cost should not be overblamed.

Further work includes further exploring of more effective guideline in determining the sizes for neighborhoods $N$ and $n$ under different noise levels and estimation of threshold parameter $\delta$ and in finding effective ways to lower the computation cost.

\section{Acknowledgment}

This research was supported by National Basic Research Program of China under Grant no. 2010CB732503.

\section{References}

[1] A. Srivastava, A. B. Lee, E. P. Simoncelli, and S. -C. Zhu, "On advances in statistical modeling of natural images," Journal of Mathematical Imaging and Vision, vol. 18, no. 1, pp. 17-33, 2003.

[2] S. Tan and L. Jiao, "A unified iterative denoising algorithm based on natural image statistical models: derivation and examples," Optics Express, vol. 16, no. 2, pp. 975-992, 2008. 
[3] R. Molina, "On the hierarchical Bayesian approach to image restoration: applications to astronomical images," IEEE Transactions on Pattern Analysis and Machine Intelligence, vol. 16, no. 11, pp. 1122-1128, 1994.

[4] G. Archer and D. M. Titterington, "On some Bayesian/ regularization methods for image restoration," IEEE Transactions on Image Processing, vol. 4, no. 7, pp. 989-995, 1995.

[5] S. Geman and D. Geman, "Stochastic relaxation, Gibbs distributions, and the bayesian restoration of images," IEEE Transactions on Pattern Analysis and Machine Intelligence, vol. 6, no. 6, pp. 721-741, 1984.

[6] N. P. Galatsanos and A. K. Katsaggelos, "Methods for choosing the regularization parameter and estimating the noise variance in image restoration and their relation," IEEE Transactions on Image Processing, vol. 1, no. 3, pp. 322-336, 1992.

[7] J. M. Bioucas-Dias and M. A. T. Figueiredo, "A new TwIST: two-step iterative shrinkage/thresholding algorithms for image restoration," IEEE Transactions on Image Processing, vol. 16, no. 12, pp. 2992-3004, 2007.

[8] Y. Wan and R. Nowak, "A Bayesian multiscale approach to joint image restoration and edge detection," in Wavelet Applications in Signal and Image Processing VII, vol. 3813 of Proceedings of SPIE, pp. 73-84, Denver, Colo, USA, July 1999.

[9] M. A. T. Figueiredo and R. D. Nowak, "An EM algorthim for wavelet-besed image restoration," IEEE Transactions on Image Processing, vol. 12, no. 8, pp. 906-916, 2003.

[10] J. M. Bioucas-Dias, "Bayesian wavelet-based image deconvolution: a GEM algorithm exploiting a class of heavy-tailed priors," IEEE Transactions on Image Processing, vol. 15, no. 4, pp. 937-951, 2006.

[11] R. Neelamani, H. Choi, and R. Baraniuk, "ForWaRD: Fourierwavelet regularized deconvolution for Ill-conditioned systems," IEEE Transactions on Signal Processing, vol. 52, no. 2, pp. 418-433, 2004.

[12] C. A. Bouman and K. Sauer, "A generalized Gaussian image model for edge-preserving MAP estimation," IEEE Transactions on Image Processing, vol. 2, no. 3, pp. 296-310, 1993.

[13] Y. Wang, J. Liu, and X. Tang, "Robust 3D face recognition by local shape difference boosting," to appear in IEEE Transactions on Pattern Analysis and Machine Intelligence.

[14] L. I. Rudin, S. Osher, and E. Fatemi, "Nonlinear total variation based noise removal algorithms," Physica D, vol. 60, no. 1-4, pp. 259-268, 1992.

[15] A. Chambolle, "An algorithm for total variation minimization and applications," Journal of Mathematical Imaging and Vision, vol. 20, no. 1-2, pp. 89-97, 2004.

[16] M. Mignotte, "A segmentation-based regularization term for image deconvolution," IEEE Transactions on Image Processing, vol. 15, no. 7, pp. 1973-1984, 2006.

[17] G. K. Chantas, N. P. Galatsanos, and A. C. Likas, "Bayesian restoration using a new nonstationary edge-preserving image prior," IEEE Transactions on Image Processing, vol. 15, no. 10, pp. 2987-2997, 2006.

[18] G. Chantas, N. Galatsanos, and A. Likas, "Bayesian image restoration based on variational inference and a product of student-t priors," in Proceedings of the 17th IEEE International Workshop on Machine Learning for Signal Processing (MLSP '07), pp. 93-98, Thessaloniki, Greece, August 2007.

[19] Y. Chen, J. Ma, Q. Feng, L. Luo, P. Shi, and W. Chen, "Nonlocal prior Bayesian tomographic reconstruction," Journal of Mathematical Imaging and Vision, vol. 30, no. 2, pp. 133-146, 2008.
[20] Y. Chen, D. Z. Gao, C. Nie, et al., "Bayesian statistical reconstruction for low-dose X-ray computed tomography using an adaptive-weighting nonlocal prior," Computerized Medical Imaging and Graphics, vol. 33, no. 7, pp. 495-500, 2009.

[21] Y. Chen, L. Hao, X. Ye, W. Chen, L. Luo, and X. Yin, "PET transmission tomography using a novel nonlocal MRF prior," Computerized Medical Imaging and Graphics, vol. 33, no. 8, pp. 623-633, 2009.

[22] J. W. Woods, S. Dravida, and R. Mediavilla, "Image estimation using doubly stochastic Gaussian random field models," IEEE Transactions on Pattern Analysis and Machine Intelligence, vol. 9, no. 2, pp. 245-253, 1987.

[23] D. F. Yu and J. A. Fessler, "Edge-preserving tomographic reconstruction with nonlocal regularization," IEEE Transactions on Medical Imaging, vol. 21, no. 2, pp. 159-173, 2002.

[24] A. Buades, B. Coll, and J.-M. Morel, "A non-local algorithm for image denoising," in Proceedings of the IEEE Computer Society Conference on Computer Vision and Pattern Recognition (CVPR '05), vol. 2, pp. 60-65, San Diego, Calif, USA, June 2005.

[25] C. A. Bouman and K. Sauer, "A unified approach to statistical tomography using coordinate descent optimization," IEEE Transactions on Image Processing, vol. 5, no. 3, pp. 480-492, 1996.

[26] S. Sotthivirat and J. A. Fessler, "Image recovery using partitioned-separable paraboloidal surrogate coordinate ascent algorithms," IEEE Transactions on Image Processing, vol. 11, no. 3, pp. 306-317, 2002.

[27] K. Lange, "Convergence of EM image reconstruction algorithms with Gibbs smoothing," IEEE Transactions on Medical Imaging, vol. 9, no. 4, pp. 439-446, 1990.

[28] W. Chen, M. Chen, and J. Zhou, "Adaptively regularized constrained total least-squares image restoration," IEEE Transactions on Image Processing, vol. 9, no. 4, pp. 588-596, 2000. 